\title{
Sub-nm Resolution, Sub-pm Precision Structure Mapping Robust to Thickness and Tilt Variations by Cepstral Analysis of Scanning Nanodiffraction 4D-STEM
}

\author{
Paul Cueva $^{1 *}$, Elliot Padget ${ }^{1}$ and David A. Muller ${ }^{1,2}$ \\ 1. School of Applied and Engineering Physics, Cornell University, Ithaca, NY, USA \\ 2. Kavli Institute at Cornell for Nanoscale Science, Ithaca, NY, USA \\ * Corresponding Author: pdc23@cornell.edu
}

Scanning nano-beam electron diffraction (NBED), obtained from 4D-STEM datasets enabled by fast, high dynamic range, and low noise pixelated detectors[1], offers a quick, precise, and high-resolution technique for structural characterization. Typical maps can be recorded in about a minute with sufficient signal to reach sub-pm precision. For sufficiently thin samples, such as 2D materials, kinematic descriptions of the diffraction patterns are sufficient for the direct determination of the full 3D structure including strain and tilt [2]. However, the nonlinear nature of dynamic diffraction complicates the analysis of samples thicker than a few nanometers. The scanning speed and spatial resolution achievable in 4D-STEM makes it difficult to implement precession schemes to reduce dynamical contrast. Here we present cepstral analysis [3] as a method to decouple the underlying lattice structure from its dynamical envelope, providing a robust, quantitative technique for the crystallography of arbitrary samples.

The patterns obtained in NBED contain the full local structural configuration, but small changes in tilt or thickness generate significant changes in intensity of the diffracted beams. Alternatively, transforming each NBED to an Exit Wave Power Cepstrum (EWPC) naturally decouples the effects of tilt and thickness from changes to the lattice structure [4]. The EWPC is a Patterson-like function showing paircorrelation peaks -i.e. distances to neighboring atoms, with crystal tilt visible as a distortion of the central peak. Figure la shows the NBED pattern of on-axis $\mathrm{PbTiO}_{3}$ and $1 \mathrm{~b}$ the corresponding EWPC pattern. A slight mistilt from a low angle grain boundary causes significant changes to the NBED pattern (Figure 1c), whereas the EWPC (Figure 1d) remains largely unaffected.

The standard approach of generating a virtual dark field image by integrating the electrons scattered to a specific angle in each NBED pattern is dominated by diffraction contrast artifacts that obscure the underlying grain contrast. Figure $2 \mathrm{a}$ demonstrates the effect of small changes in tilt, and Figure $2 \mathrm{~b}$ illustrates thickness fringes within a grain. The cepstral dark field analysis of the same 4D-STEM data set, obtained by integrating the peaks in the EWPC, is robust to diffraction artifacts and shows clearly interpretable grain contrast (Figure 2c,d).

Finally, 4D-STEM strain measurements, whether by atom tracking or diffraction techniques, usually require the sample to be on axis [5]. Instead, by measuring the changes of peak positions in the EWPC we calculate the strain in arbitrarily oriented nanoparticles. Though the PtCo core-shell nanoparticle in Figure 3a is far off zone-axis (Figure 3b), Figure 3c,d shows the lattice expansion of the Pt shell with sub-pm precision and sub-nm resolution. This technique allows for high-throughput strain mapping of randomly configured nanoparticles. We discuss the physical motivation for the efficacy of EWPC analysis and demonstrate structural characterization in a wide range of applications including ferroelectrics and catalysis [6].

References:

[1] M Tate, et al., Microscopy and Microanalysis 22 (2016), pp. 237-249.

[2] Y. Han, et al., Nano Letters, 18 (2018), pp. 3746-3751. 
[3] JK Lee, et al., Pattern Recognition, 2610 (1993), pp. 1579-1592.

[4] P Cueva, E Padget and DA Muller, Microscopy and Microanalysis 24S1 (2018), pp. 490-491.

[5] VB Ozdol, et al., Applied Physics Letters 106 (2015), 253107.

[6] PC was supported by the Center for Bright Beams, an NSF STC (PHY-1549132). EP supported by

DOE EERE (DE-EE0007271). This work made use of the Cornell Center for Materials Research Shared

Facilities, an NSF MRSEC (DMR-1719875 and NSF-MRI-1429155).
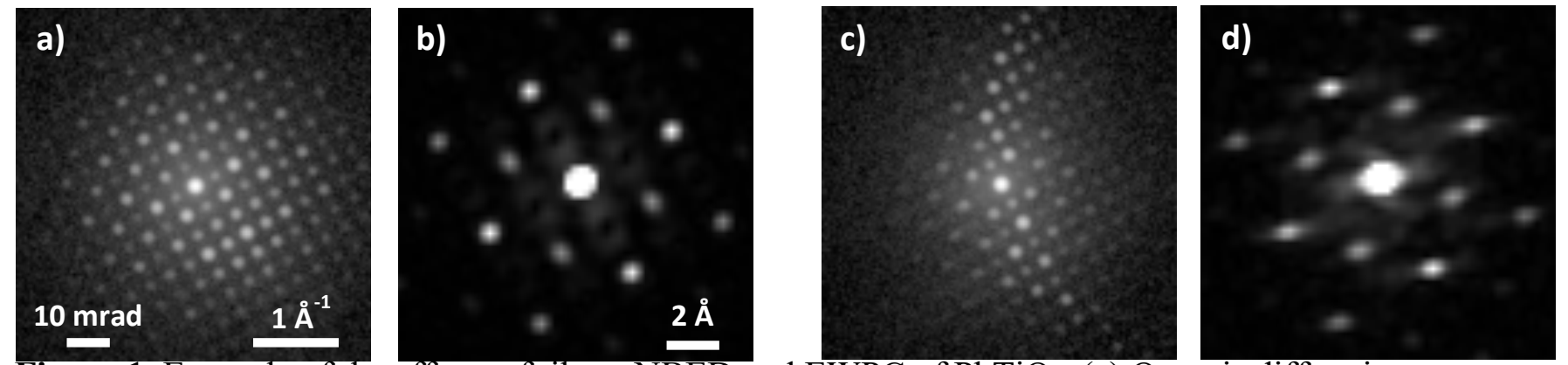

Figure 1. Example of the effects of tilt on NBED and EWPC of $\mathrm{PbTiO}_{3}$. (a) On-axis diffraction pattern and (b) the corresponding EWPC demonstrate clear points of symmetry. (c) Slight mistilts from a low angle grain boundary greatly affect the contrast of diffraction spots, (d) whereas the corresponding EWPC only suffers low frequency distortions.

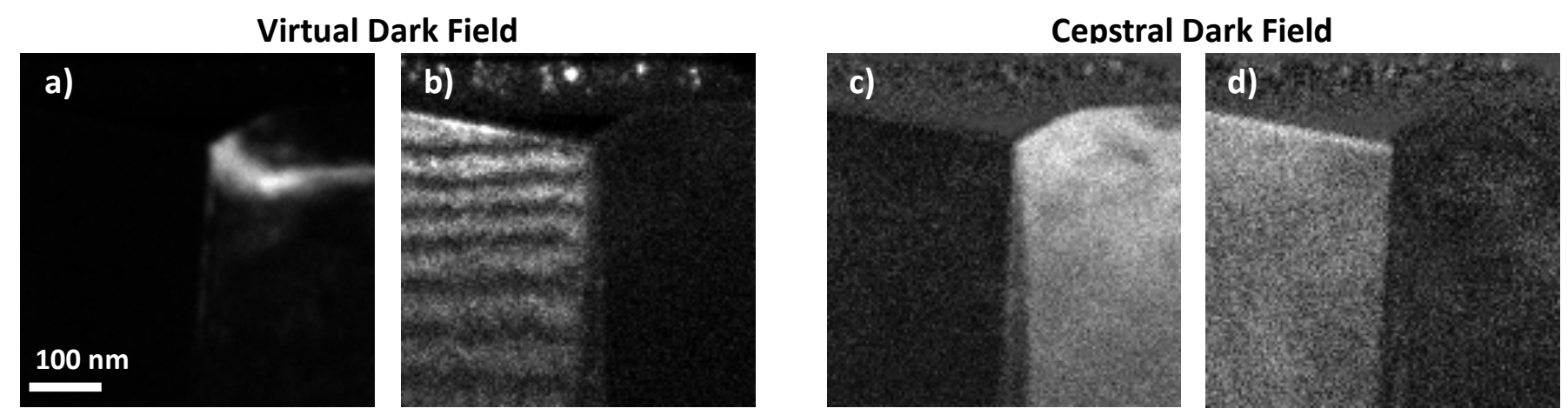

Figure 2. Comparison of dark field techniques for grain determination in polycrystalline, off-axis $\mathrm{Nb}_{3} \mathrm{Sn}$. (a,b) Conventional virtual dark field approaches are dominated by tilt contrast and thickness fringes. (c,d) Cepstral dark field provides clear grain contrast.
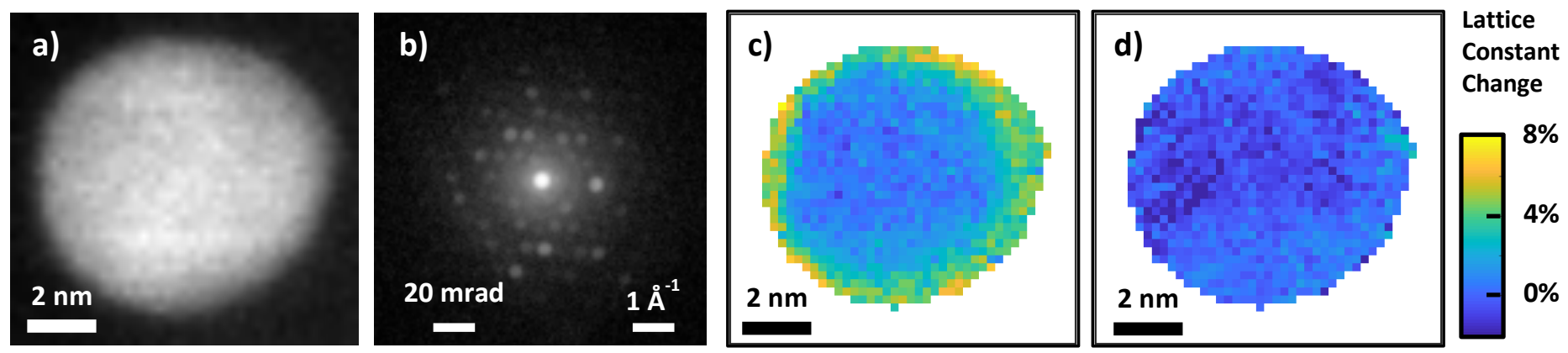

Figure 3. Strain map for a PtCo alloy fuel cell catalyst nanoparticle with Co leached from near the surface to form a compressively strained Pt shell. (a) Virtual annular dark field image and (b) nanobeam electron diffraction pattern from 4D scanning diffraction map of an arbitrarily oriented nanoparticle. Quantitative maps of changes in the relative lattice constant show the major (c) and minor (d) axes of the distortion field due to the radial expansion of the compressed Pt shell. 